Open Access

\title{
Reliable reference genes for expression analysis of proliferating and adipogenically differentiating human adipose stromal cells
}

\author{
Claudia Krautgasser ${ }^{1 \dagger}$, Markus Mandl $^{1 \dagger}$, Florian M. Hatzmann ${ }^{1}$, Petra Waldegger ${ }^{1}$, Monika Mattesich ${ }^{2}$
} and Werner Zwerschke ${ }^{1 *}$

\footnotetext{
* Correspondence: werner. zwerschke@uibk.ac.at

${ }^{+}$Claudia Krautgasser and Markus Mandl have equal contribution

${ }^{1}$ Division of Cell Metabolism and Differentiation Research, Institute for Biomedical Aging Research, University of Innsbruck, Rennweg 10, A-6020 Innsbruck, Austria Full list of author information is available at the end of the article
}

\begin{abstract}
Background: The proliferation and adipogenic differentiation of adipose stromal cells (ASCs) are complex processes comprising major phenotypical alterations driven by up- and downregulation of hundreds of genes. Quantitative RT-PCR can be employed to measure relative changes in the expression of a gene of interest. This approach requires constitutively expressed reference genes for normalization to counteract inter-sample variations due to differences in RNA quality and quantity. Thus, a careful validation of quantitative RT-PCR reference genes is needed to accurately measure fluctuations in the expression of genes. Here, we evaluated candidate reference genes applicable for quantitative RT-PCR analysis of gene expression during proliferation and adipogenesis of human ASCs with the immunophenotype DLK $1^{+} / \mathrm{CD} 34^{+} / \mathrm{CD} 90^{+} / \mathrm{CD} 105^{+} / \mathrm{CD} 45^{-} / \mathrm{CD} 31^{-}$.
\end{abstract}

Methods: We evaluated the applicability of 10 candidate reference genes (GAPDH, TBP, RPS18, EF1A, TFRC, GUSB, PSMD5, CCNA2, LMNA and MRPL19) using NormFinder, geNorm and BestKeeper software.

Results: The results indicate that EFIA and MRPL19 are the most reliable reference genes for quantitative RT-PCR analysis of proliferating ASCs. PSMD5 serves as the most reliable endogenous control in adipogenesis. CCNA2 and LMNA were among the least consistent genes.

Conclusions: Applying these findings for future gene expression analyses will help elucidate ASC biology.

Keywords: Adipose stromal cells, Adipose stem cells, Adipogenesis, Proliferation, Differentiation, Reference gene

\section{Background}

Adipose stromal cells (ASCs) are a major reservoir of adipocyte precursors in adipose tissues [1, 2]. Their heterogeneous population contains stem and progenitor cells, which are essential for adipose tissue development, regeneration and homeostasis [3]. Thus, ASCs are crucial for the maintenance of adipose tissue functions. They are also a viable source of material for cell-based therapies in regenerative medicine [4].

The large population of human ASCs with the immunophenotype DLK1 $1^{+} / \mathrm{CD} 34^{+} /$ $\mathrm{CD} 90^{+} / \mathrm{CD} 105^{+} / \mathrm{CD} 45^{-} / \mathrm{CD} 31^{-}$consists of adipose progenitor cells with high

(c) The Author(s). 2019 Open Access This article is distributed under the terms of the Creative Commons Attribution 4.0 International License (http://creativecommons.org/licenses/by/4.0/), which permits unrestricted use, distribution, and reproduction in any medium, provided you give appropriate credit to the original author(s) and the source, provide a link to the Creative Commons license, and indicate if changes were made. The Creative Commons Public Domain Dedication waiver (http://creativecommons.org/ publicdomain/zero/1.0/) applies to the data made available in this article, unless otherwise stated. 
proliferative and adipogenic potential $[5,6]$. Proliferation is a highly dynamic process involving many changes in gene expression occurring in response to extracellular signals and as a function of cell cycle progression regulated by specific cyclin-dependent kinase activities [7]. The differentiation of ASCs into adipocytes, also referred to as adipogenesis, gives rise to a new cell type [8]. A cascade of transcriptional regulators orchestrates adipogenesis. The expression of the adipogenic transcription factor peroxisome proliferator-activated receptor $\gamma 2$ (PPAR $\gamma 2$ ), which is both required and sufficient to drive adipogenesis, is a key event in the induction of the adipogenic differentiation program $[9,10]$. The function of PPAR $\gamma 2$ is tightly linked to members of the CCAAT/enhancer-binding protein (C/EBP) family of transcription factors [11]. The functional interaction of these factors induces changes in the expression of a large number of genes, leading to distinct morphological and biochemical alterations and eventually to the generation of adipocytes [12]. To accurately analyze the changes in gene expression during both proliferation and adipogenic differentiation of ASCs, appropriate endogenous controls are needed.

Valid reference genes are crucial for reliable gene expression analyses using quantitative RT-PCR $[13,14]$. They serve as internal controls, allowing normalization, which counteracts inter-sample variations due to differences in RNA quantity and quality [13, 15]. State-of-the-art quantitative RT-PCR is essential for the validation of appropriate reference genes $[16,17]$.

A constitutive expression pattern qualifies a certain gene to act as internal control $[15,16,18]$. Commonly used reference genes include those coding for metabolic enzymes, such as glyceraldehyde-3-phosphate dehydrogenase (GAPDH), or components of the cytoskeleton, such as $\beta$-actin $(A C T B)$ and $\beta$-2-microglobulin $(B 2 M)[16,17]$. The $18 \mathrm{~S}$ ribosomal RNA is also considered to be a reliable reference in quantitative RT-PCR experiments [19]. However, it is known that the expressions of genes coding for metabolic enzymes [20] and cytoskeleton components [21] are regulated and dependent on the physiological conditions or cellular state. In fact, no single gene exists which is constitutively expressed in all cell types under all experimental conditions $[15,18]$.

Thus, a careful validation of reference genes for given applications is mandatory. There is still a need for appropriate endogenous controls for quantitative RT-PCR analysis to cope with the strong fluctuations in gene expression during the complex processes of ASC proliferation and adipocyte differentiation. This study aimed to evaluate candidate reference genes reliable for quantitative RT-PCR analysis of proliferating and differentiating human ASCs to enable future elucidation of ASC biology.

\section{Methods}

\section{Study design}

We aimed to identify appropriate quantitative RT-PCR reference genes for the analysis of gene expression in the course of proliferation and adipogenesis of human ASCs.

\section{Isolation and cultivation of ASCs}

Human ASCs were isolated from abdominal subcutaneous white adipose tissue (sWAT) samples obtained from four females undergoing routine elective plastic abdominal surgery at the Institute for Plastic and Reconstructive Surgery of the Medical University of 
Innsbruck. All patients gave their informed written consent. The study protocol was approved by the Ethics Committee of the Medical University of Innsbruck and complies with the Helsinki Declaration. The characteristics of the donors are given in Additional file 1: Table S1. Isolation and cultivation of ASCs was performed as described previously [6]. ASCs were maintained in PM4 medium, which is DMEM/F-12 medium (1:1) with HEPES and l-glutamine (Gibco) containing $33 \mu \mathrm{M}$ biotin, $17 \mu \mathrm{M}$ pantothenate $20 \mu \mathrm{g} / \mathrm{ml}$ ciprofloxacin, 2.5\% FCS, $10 \mathrm{ng} / \mathrm{ml}$ EGF, $1 \mathrm{ng} / \mathrm{ml} \mathrm{bFGF}$ and $500 \mathrm{ng} / \mathrm{ml}$ insulin. The ASCs were split at $70 \%$ confluence. Passage 3 ASCs were used for the whole study.

\section{Adipogenic differentiation}

Adipogenesis was induced as described previously [6]. ASCs were expanded until confluence in PM4 medium and serum-starved for another $48 \mathrm{~h}$ in serum-free ASC medium consisting of DMEM/F-12 medium (1:1) with HEPES and l-glutamine (Gibco) containing $33 \mu \mathrm{M}$ biotin, $17 \mu \mathrm{M}$ pantothenate and $20 \mu \mathrm{g} / \mathrm{ml}$ ciprofloxacin. Adipogenesis was induced by the addition of differentiation medium, which was ASC medium containing $0.2 \mu \mathrm{M}$ insulin, $0.5 \mathrm{mM}$ IBMX, $0.25 \mu \mathrm{M}$ dexamethasone, $2.5 \%$ FCS and $10 \mu \mathrm{g} / \mathrm{ml}$ transferrin. After 3 days, the medium was changed, and the cells were cultivated until day 14 in differentiation medium without IBMX. Cell extracts for western blot and gene expression analysis were taken at defined time points before and after induction of adipogenic differentiation. Oil-Red-O staining was performed to visualize lipid droplets of differentiated adipocytes. Briefly, cells were fixed in a $4 \%$ paraformaldehyde-PBS mixture for $30 \mathrm{~min}$, washed with PBS, stained with $0.5 \%$ Oil-Red-O in a 60:40 isopropanol:water mix for $1 \mathrm{~h}$, and finally washed with $\mathrm{H}_{2} \mathrm{O}$.

\section{Proliferation monitoring}

For the proliferation assays, 6-well plates were seeded with 60,000 cells/well. The cells were cultivated in PM4 medium containing 2.5\% FCS (low mitogenic medium) and PM4 medium containing 10\% FCS (high mitogenic medium) in the same experiment. Cell numbers were counted at defined time points using a Neubauer Chamber.

\section{Flow cytometry analysis}

Fluorescence-activated cell sorting (FACS) was used to characterize the immunophenotype of human ASCs. $1 \times 10^{5}$ cells per sample were simultaneously fixed and permeabilized using BD Cytofix/Cytoperm solution to prepare the cells for intracellular and surface staining. The cells were subjected to immunofluorescence staining using a panel of mouse monoclonal antibodies (BD Pharmingen): CD34-PE-Cy7 (\#560710), CD105-PerCP-Cy5.5 (\#560819), CD90-PE (\#561970), CD45RA-FITC (\#556626) and CD31-FITC (\#555445). For DLK1 a primary rat monoclonal anti-human DLK1/PREF1 antibody (Adipogen, AG-25A-0091) along with the anti-rat-APC antibody (BD Pharmingen, \#551019) was used. The labeled cells were measured using a FACS Canto II (BD Biosciences) and the data were analyzed using Flowing Software (http://www.flowingsoftware.com).

\section{RNA isolation and quantitative RT-PCR}

Isolation of RNA was done using the RNeasy Plus Micro Kit (Qiagen, \#74034) according to the manufacturer's instructions. Briefly, cells were washed with PBS and lysed 
immediately with $350 \mu \mathrm{l}$ RLT buffer per well. Genomic DNA (gDNA) was removed from the cell lysate by filtration through a gDNA Eliminator spin column (Qiagen). Subsequently, one volume 70\% ethanol was added and the sample transferred onto the RNeasy MinElute binding column. Bound RNA was washed with RW1 buffer, RPE buffer and $80 \%$ ethanol. After elution, the yield and purity of the isolated RNA was determined spectrophotometrically. RNA integrity was confirmed using agarose gel electrophoresis.

For reverse transcription of RNA ( $1.5 \mu \mathrm{g}$ per sample), we used the First Strand cDNA Synthesis Kit (Thermo Scientific, \#K1622) according to the manufacturer's guidelines. Subsequently, cDNA was diluted 1:15 and an aliquot of $5 \mu$ was used for quantitative RT-PCR analysis.

Gene expression was measured with a LightCycler 480 (Roche) instrument using SYBR green chemistry and confirmed using corresponding non-template controls. Genomic DNA contamination was excluded using appropriate mock reverse-transcriptase controls. Primer were designed using QuantPrime [22] (http://quantprime.mpimp-golm.mpg.de/) and are given in Additional file 1: Table S3. Primer specificity was verified via melting curve analysis. The number of biological replicates (i.e., donors) per group was $n=3$ (proliferation) and $n=4$ (adipogenesis). All samples were measured in duplicate.

\section{Western blot analysis}

ASCs were harvested in SDS lysis buffer and sonicated as described previously [6]. The protein concentration of the samples was determined with the Combat-Able Protein Assay Preparation Reagent Set (Thermo Scientific, \#23215) and the Pierce BCA Protein Assay Kit (Thermo Scientific, \#23227). Samples (10 $\mu \mathrm{g}$ total protein) were separated on $10 \%$ SDS-polyacrylamide gel, blotted onto a PVDF membrane and probed with polyclonal anti-perilipin antibodies (Cell Signaling Technology, \#9349). To ensure equal loading and blotting, membranes were probed with an anti- $\beta$-actin antibody (Sigma Aldrich, AC15). Goat anti-rabbit IgG-HRP (DAKO) and anti-mouse IgG-HRP (Promega) served as secondary antibodies. Signals were detected using a chemiluminescence detection system.

\section{Software packages for quantitative RT-PCR analysis}

Quantitative RT-PCR results were analyzed using three software packages, NormFinder, geNorm and BestKeeper, according to the developer's instructions. The NormFinder algorithm was described by Andersen et al. [18] (software download: https://moma.dk/ normfinder-software). The principles of the geNorm program were described by Vandesompele et al. [23] (software download: https://genorm.cmgg.be/). The BestKeeper software was described by Pfaffl et al. [24] (software download: http:// www.gene-quantification.de/bestkeeper.html). Candidate reference genes were ranked based on the relevant algorithm.

\section{Statistics}

Statistical analysis was performed using GraphPad Prism 5 (GraphPad) software. The number of different donors per group was $n=3$ (proliferation) and $n=4$ (adipogenesis). 
Comparison of two groups was achieved using Student's $t$-test. $p \leq 0.05$ was considered statistically significant.

\section{Results}

\section{Proliferation and adipogenic differentiation of human ASCs}

We evaluated candidate reference genes for quantitative RT-PCR-based gene expression studies of proliferating and differentiating human ASCs. First, we isolated ASCs from fresh abdominal sWAT samples obtained by incision from four female donors undergoing elective plastic abdominal surgery (Additional file 1: Table S1). The cells were stored in liquid nitrogen. To determine the purity of the ASC population, the cells were thawed and grown to passage 3. Afterwards the cells were permeabilized and subjected to multi-parameter detection FACS analysis using antibodies against established ASC marker proteins $[6,25]$. The vast majority of the cells showed the characteristic ASC immunophenotype, $\mathrm{DLK} 1^{+} / \mathrm{CD} 34^{+} / \mathrm{CD} 90^{+} / \mathrm{CD} 105^{+} / \mathrm{CD} 45^{-} / \mathrm{CD} 31^{-}$(Fig. 1a), which is expected for permeabilized passage 3 ASCs $[6,25]$.

The ASCs were then cultured in a low mitogenic medium (PM4 medium containing $2.5 \%$ FCS) and in a high mitogenic medium (PM4 medium containing 10\% FCS).

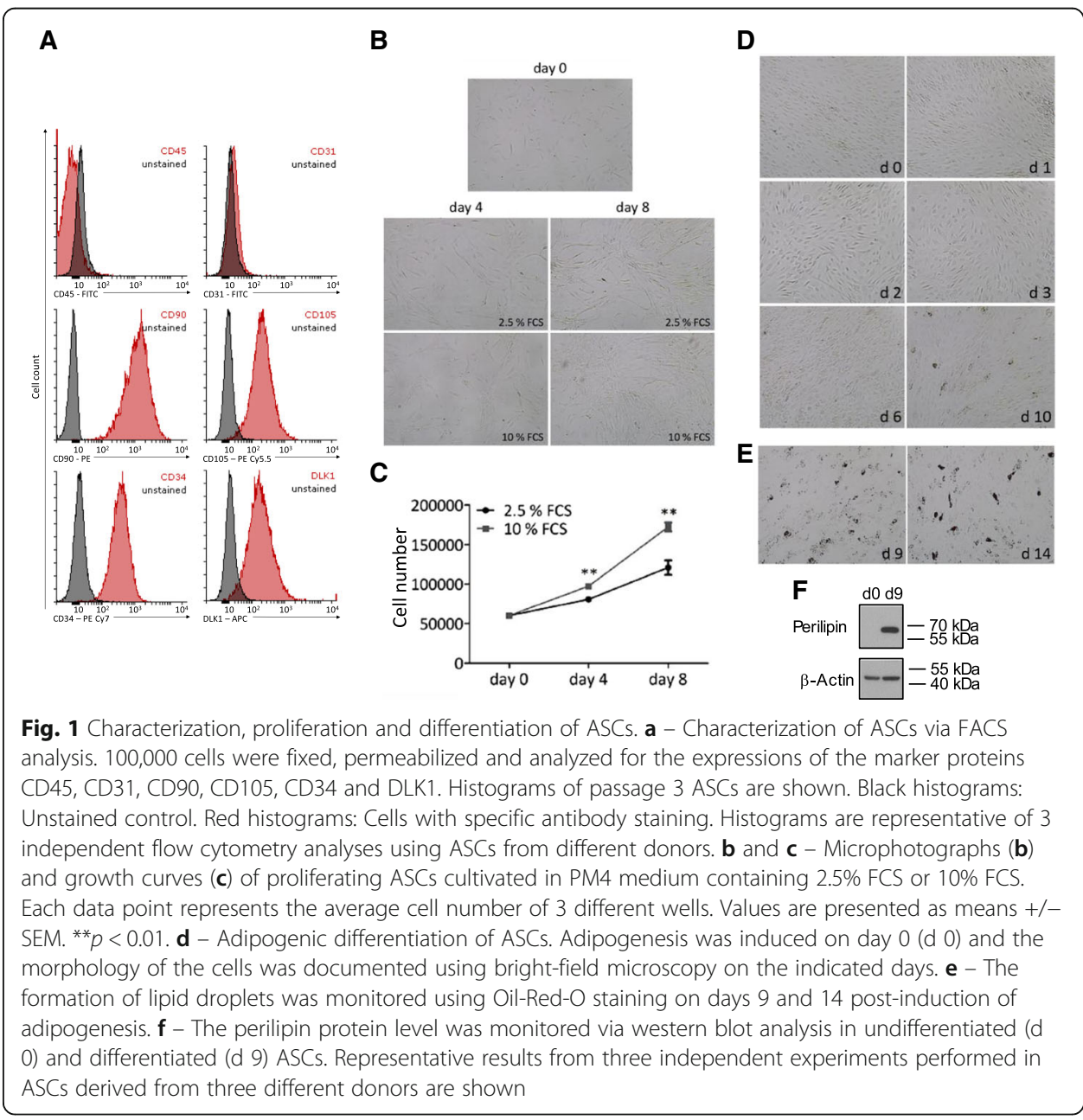


Proliferation was monitored by counting the ASC numbers on the indicated days (Fig. $1 \mathrm{~b}$ and $\mathrm{c}$ ). As expected, ASCs grown in high mitogenic medium showed a significantly higher proliferation rate compared to cells grown in low mitogenic medium.

For adipogenic differentiation, ASCs were grown to density arrest and starved in serum-free medium. Induction of adipogenesis by a hormone cocktail led to the characteristic morphological transformation of ASCs from a fibroblast-like morphology to rounded cells in the course of the first $72 \mathrm{~h}$ after induction (Fig. 1d). This is a hallmark of adipogenesis [26]. Differentiation was confirmed by detection of intracellular fat droplets (Fig. 1e) and the adipocyte-specific protein perilipin (Fig. 1f). The full western blot analysis is shown in Additional file 2: Figure S1.

\section{Selection and expression levels of reference genes}

For quantitative RT-PCR analysis, total RNA was isolated from proliferating ASCs (3 donors) and from ASCs 0, 1, 2, 3, 6 and 10 days after induction of adipogenesis (4 donors). The yield ranged from 2 to $10 \mu$ g with a mean purity ratio (A260/A280) of 2.0.

We selected several candidate reference genes (Additional file 1: Table S2) to find the most reliable ones for RNA expression analysis in proliferating and differentiating ASCs. Standard curves for reference genes were processed based on proliferating ASCs whereas standards for adipogenic marker genes were performed on ASCs three days after the induction of adipogenesis (Additional file 3: Figure S2 and Additional file 4: Figure S3).

Quantitative RT-PCR for standards was performed using the classical 10-fold serial dilution method. The efficiencies (E) of reference and target gene primer sets had mean values of $101.9+/-2.81 \%$ and $103.9+/-3.80 \%$, respectively (Additional file 4: Table S3). To confirm a specific PCR amplification, gel electrophoresis was performed. It showed only one PCR product at the predicted size (Fig. 2a). Additionally, melting curve analysis exhibited one clear peak for each primer pair (data not shown).

The tested reference genes showed various expression levels (Fig. 2b and c). To evaluate if the increased SDs within these four groups refer to significant outliers we performed Grubbs' test, which detects outliers in a given data set and defines their significance. No significant outlier was detected with the threshold at $p \leq 0.05$. Therefore, all samples were included for further analysis.

\section{Evaluation of appropriate reference genes for ASC proliferation and differentiation}

The candidate reference genes selected in this study encode proteins in different functional classes so the chance that genes might be co-regulated is low [18]. To select optimal reference genes for ASC proliferation and differentiation, three different mathematical approaches (GeNorm, NormFinder and BestKeeper) were used:

GeNorm analysis ranks candidate reference genes with their lowest expression stability value (M-value) up to a threshold of 0.5 . Genes with values exceeding 0.5 are considered unstable [27], although in heterogeneous cell populations, an M-value of 1.0 can also be accepted [27]. ASCs undergoing the differentiation process cannot be regarded as a homogeneous cell population compared to cycling ASCs. Therefore, the threshold was set to 0.5 for proliferating cells and 1.0 for differentiating cells. The M-values generated using the GeNorm software are presented in Fig. 3a and b. 


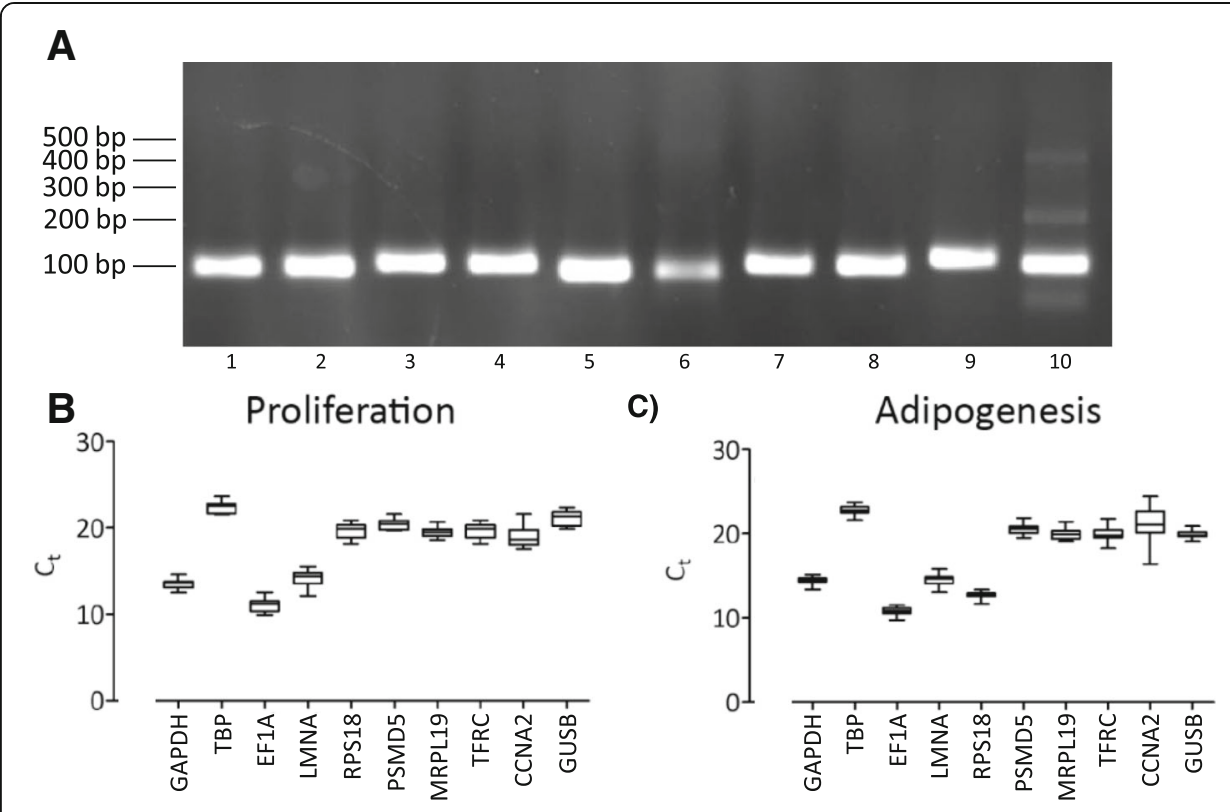

Fig. 2 Primer specificity and average raw cycle thresholds. a - The amplification specificity of all candidate reference gene primer sets. CDNA was isolated from undifferentiated human ASCs. Lanes 1-10: GAPDH, TBP, EF1A, LMNA, RPS18, PSMD5, MRPL19, TCRF, CCNA2 and GUSB. B and C - Raw quantitative PCR C Talues for candidate reference genes during proliferation (b) and adipogenesis (c). Each gene was amplified in 15 (proliferation) or 24 (adipogenesis) different biological samples in duplicates. Values are presented as means +/- SEM

The NormFinder algorithm calculates the stability value of each gene. Based on this analysis, the use of two candidate genes with the lowest stability is recommended (threshold 0.15) [18]. As shown in Fig. 3c, candidate genes (MRPL19, TBP, EF1A, $P M S D 5$ and $G A P D H)$ meet the criteria defined by the normalization factor cut off value 0.15 for proliferating ASCs. NormFinder analysis revealed MRPL19 and TBP to be the best combination of reference genes for proliferating ASCs (stability value 0.075). However, the stability values of candidate genes tested in differentiating ASCs failed to remain below the threshold of 0.15 (Fig. 3d). As mentioned above, these higher values might be due to the heterogeneity of differentiating cells. However, the combination of PSMD5 and TBP changed the stability value to an acceptable number of 0.122 .

BestKeeper analysis step-wisely excludes unsuitable candidate reference genes. After descriptive statistical analysis for each reference gene, candidates with a standard deviation above 1.0 are immediately excluded. Subsequently, pair-wise correlation analysis is performed to calculate the Pearson correlation co-efficient $\mathrm{R}$ for every reference gene. High $R$ values are considered to indicate a stable gene expression pattern [24]. Analysis of $\mathrm{C}_{\mathrm{T}}$ values of all candidate genes in proliferating ASCs revealed a SD (standard deviation) below 1.0 (data not shown). CCNA2 was excluded from further calculations due to its high SD (0.89). Further analysis showed a strong correlation for all candidate genes $(0.977<\mathrm{R}<0.741$; Fig. 3e). When we repeated BestKeeper analysis with the three most appropriate genes, MRPL19, GUSB and EF1A, the correlation even increased $(0.985<\mathrm{R}<0.987$, Additional file 1: Table S4). After excluding CCNA2 (SD = $1.5)$, candidate reference genes in adipogenic ASCs showed a rather weak correlation $(0.920<\mathrm{R}<0.437$, Fig. 3f). However, examination of the three best candidates (PSMD5, 

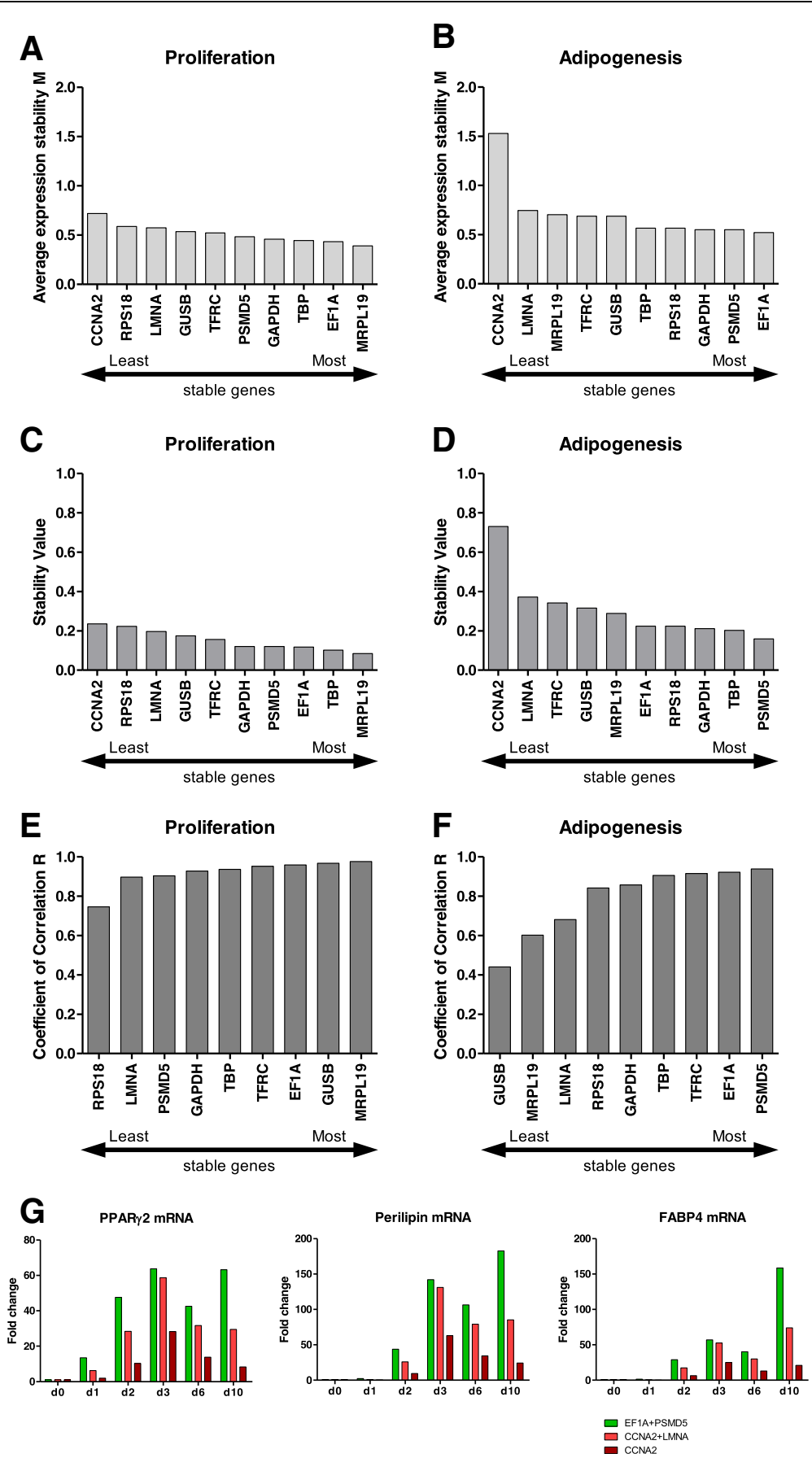

Fig. 3 Analysis and ranking of candidate gene expression to determine the most stable reference genes in proliferation and adipogenesis. $\mathbf{a}$ and $\mathbf{b}$ - GeNorm analysis showing the stability value $\mathbf{M}$ of candidate reference genes in proliferating (a) and differentiating (b) ASCs. Lower values indicate more stable genes, higher values indicate less stable genes. $\mathbf{c}$ and $\mathbf{d}$ - NormFinder analysis showing the most stable reference genes in proliferating (c) and differentiating (d) ASCs. Lower values indicate more stable genes, higher values indicate less stable genes. $\mathbf{e}$ and $\mathbf{f}$ - BestKeeper analysis showing the most stable reference genes (based on their Pearson correlation coefficient) for proliferation (e) and differentiation (f). Higher values indicate more stable genes, lower values indicate less stable genes. $p<0.001$ (exceptions: proliferation: RPS18 $p=0.002$; adipogenesis: GUSB $p=0.03$, MRPL19 $p=0.003$ ). $\mathbf{g}$ - Effects of appropriate (green) and inappropriate (red) reference genes on the relative expressions of adipogenic marker genes in the course of adipogenesis are shown 
EF1A and TFRC) revealed a strong correlation between these genes $(0.969<\mathrm{R}<0.935$, Additional file 1: Table S4).

The impact of different reference genes on the expressions of genes of interest (GOIs) was evaluated in differentiating ASCs. Representative time-course experiments using the combinations EF1A + PSMD5, CCNA2 + LMNA and only CCNA2 as the reference gene(s) are shown in Fig. 3g. It is clear that the selection of the reference gene(s) has considerable influence on the measurement of GOI expression.

\section{Discussion}

Cell cycle progression and differentiation of ASCs into mature adipocytes are highly orchestrated and associated with major changes in the gene expression pattern [7, 8, 11]. To measure transcriptional changes during these processes, reliable approaches are required [28].

Quantitative RT-PCR is an established and highly sensitive technique to measure the expression of a gene of interest [29]. Absolute and relative quantitation of gene expression are possible with this technique. The first approach requires a costly standard curve to determine the number of transcripts present in the sample, while the latter depends on appropriate reference genes for relative quantitation of gene expression [16, 17]. In this study, we combined the NormFinder, GeNorm and BestKeeper software packages $[18,23,24]$ to define new reference genes for the comparison of gene expression in proliferating and adipogenic differentiating ASCs. Our results demonstrate the feasibility of this combinatorial approach. Similar results were obtained with all three programs.

EF1A and MRPL19 were identified as the most stably expressed genes in the proliferating ASCs. The three algorithms identified PSMD5 as the most stably expressed gene in adipogenically differentiating ASCs. EF1A was ranked by GeNorm and BestKeeper to be among the most stable reference genes for adipogenesis (Table 1). Commonly used endogenous control genes such as GAPDH showed an intermediate stability valuated during proliferation and adipogenic differentiation.

Our results underscore the context-dependent expression of reference genes and the requirement to find the most appropriate one(s) for given experimental conditions. Our findings are in agreement with those of other studies that identified EF1A and

Table 1 Ranking of reference genes

\begin{tabular}{|c|c|c|c|c|c|c|}
\hline \multirow[b]{2}{*}{ Rank } & \multicolumn{3}{|c|}{ Proliferation } & \multicolumn{3}{|c|}{ Adipogenesis } \\
\hline & GeNorm & NormFinder & BestKeeper & GeNorm & NormFinder & BestKeeper \\
\hline 1 (most stable) & MRPL19 & MRPL19 & MRPL19 & EF1A & PSMD5 & PSMD5 \\
\hline 2 & EF1A & TBP & GUSB & PSMD5 & TBP & EF1A \\
\hline 3 & TBP & EF1A & EF1A & GAPDH & GAPDH & TFRC \\
\hline 4 & GAPDH & PSMD5 & TCRF & RPS18 & RPS18 & TBP \\
\hline 5 & PSMD5 & GAPDH & TBP & TBP & EF1A & GAPDH \\
\hline 6 & TFRC & TFRC & GAPDH & GUSB & MRPL19 & RPS18 \\
\hline 7 & GUSB & GUSB & PSMD5 & TFRC & GUSB & LMNA \\
\hline 8 & LMNA & LMNA & LMNA & MRPL19 & TFRC & MRPL19 \\
\hline 9 & RPS18 & RPS18 & RPS18 & LMNA & LMNA & GUSB \\
\hline 10 (least stable) & CCNA2 & CCNA2 & & CCNA2 & CCNA2 & \\
\hline
\end{tabular}


TBP as useful endogenous controls to analyze gene expression in differentiating mesenchymal stem cells $[13,28]$. The experimental strategy of our study is a straightforward way to identify novel reference genes for quantitative RT-PCR analysis of proliferating and differentiating ASCs. This strategy has also been applied to mesenchymal stem cells of human [13] and rat origin [30].

\section{Conclusions}

Our study identified EF1A, MRPL19 and PSMD5 as new quantitative RT-PCR reference genes suitable for measuring changes in gene expression during proliferation and adipogenesis of human $\mathrm{DLK} 1^{+} / \mathrm{CD} 34^{+} / \mathrm{CD} 90^{+} / \mathrm{CD} 105^{+} / \mathrm{CD} 45^{-} / \mathrm{CD} 31^{-}$ASCs. The data suggest that EF1A and MRPL19 are the most reliable reference genes for quantitative RT-PCR analysis of proliferating ASCs with that immunophenotype and PSMD5 serves as the best endogenous control for gene expression analysis in the course of adipogenic differentiation of these cells. Applying these findings in future experiments will help elucidate ASC biology.

\section{Additional files}

Additional file 1: Table S1. Donor characteristics. All donors were healthy formerly obese Caucasian women, who underwent routine abdominoplasty. None of the women suffered from diabetes, liver, renal or other severe metabolic diseases. None of the women had cancer. Clinical and anthropometric parameters are indicated. Table S2. Selected candidate reference genes. Table S3. Primer sequences. Table S4. Results of BestKeeper analysis. (DOCX $30 \mathrm{~kb}$ )

Additional file 2: Figure S1. Detection of perilipin via western blot analysis. A - SDS-PAGE was carried out as described in the Methods section to separate proteins in cellular lysates by molecular weight. A pre-stained molecular weight marker (M) was included (Thermo Scientific, \#26616). After gel electrophoreses, the proteins were electro-blotted onto one polyvinyl-difluoride (PVDF) membrane. The transfer of pre-stained marker bands indicated a successful western blot procedure. B - Immobilized PVDF membrane-bound proteins were probed using a specific antibody against perilipin (Cell Signaling Technology, \#9349) followed by incubation with an appropriate horseradish peroxidase-conjugated (HRP-conjugated) secondary antibody (goat anti-rabbit IgG-HRP, DAKO). Signal development was achieved by applying the enhanced chemo-luminescence (ECL) substrate. The generated light signal was detected by exposure of the PVDF membrane to a Xray film. Marker bands were manually transferred onto the X-ray film by adjusting the PVDF membrane and the X-ray film according to specific marks located in the film cassette. The corresponding X-ray film to the PVDF membrane shown in (A) is presented. $\mathbf{C}$ - To ensure equal loading, the same PVDF membrane was re-probed using a specific anti- $\beta$-actin antibody (Sigma Aldrich, AC15) followed by incubation with an HRP-conjugated secondary antibody (anti-mouse lgG-HRP). After applying the ECL substrate, the membrane was exposed to an X-ray film. The corresponding X-ray film to the PVDF membrane shown in (A) is presented. The molecular weight is given in kilo Daltons (kDa). (PDF 99 kb)

Additional file 3: Figure S2. Standard curves for reference genes. (PDF $446 \mathrm{~kb}$ )

Additional file 4: Figure S3. Standard curves for target genes. (PDF $81 \mathrm{~kb}$ )

\section{Abbreviations}

ASCs: Adipose stromal cells; C/EBP: CCAAT/enhancer-binding protein; CCNA2: Cyclin A2; DLK-1: Delta-like protein 1; EF1A: Elongation factor alpha; EGF: Epidermal growth factor; FCS: Fetal Calf Serum; FGF: Fibroblast growth factor; GAPDH: Glyceraldehyde-3-phosphate dehydrogenase; gDNA: Genomic DNA; GOI: Gene of interest;

GUSB: Glucuronidase beta; LMNA: Lamin A; MRPL19: Mitochondrial ribosomal protein 19; PPAR 2: Peroxisome proliferator-activated receptor Y2; PSMD5: Proteasome 265 subunit, non-ATPase 5; RPS18: Ribosomal protein S18; RTPCR: Reverse transcription polymerase chain reaction; SD: Standard deviation; SEM: Standard error of the mean; SVF: Stromal vascular fraction; sWAT: Subcutaneous white adipose tissue; TBP: TATA-box binding protein; TFRC: Transferrin receptor

\section{Acknowledgements}

Not applicable.

Funding

This study was supported by intramural funding from the University of Innsbruck. 


\section{Authors' contributions}

CK, MaM and WZ designed the experiments. CK, MaM, FH and PW performed the experiments. CK, PW, FH, MaM, MoM and WZ analyzed the data. MoM performed the surgeries and contributed adipose tissue biopsies. CK, MaM and WZ wrote the manuscript. All authors read and approved the final manuscript.

\section{Ethics approval and consent to participate}

Subcutaneous white adipose tissue (sWAT) samples were taken from women undergoing routine abdominoplasty at the Institute for Plastic and Reconstructive Surgery of the Medical University of Innsbruck. All patients gave their informed written consent. The study protocol was approved by the Ethics Committee (Medical University of Innsbruck) according to the Declaration of Helsinki.

\section{Consent for publication}

All authors gave their consent.

\section{Competing interests}

The authors declare that they have no competing interests.

\section{Publisher's Note}

Springer Nature remains neutral with regard to jurisdictional claims in published maps and institutional affiliations.

\section{Author details}

${ }^{1}$ Division of Cell Metabolism and Differentiation Research, Institute for Biomedical Aging Research, University of Innsbruck, Rennweg 10, A-6020 Innsbruck, Austria. ${ }^{2}$ Department of Plastic and Reconstructive Surgery, Innsbruck Medical University, Anichstraße 35, A-6020 Innsbruck, Austria.

Received: 3 October 2018 Accepted: 11 February 2019

Published online: 15 February 2019

\section{References}

1. Rodeheffer MS, Birsoy K, Friedman JM. Identification of white adipocyte progenitor cells in vivo. Cell. 2008;135(2):240-9.

2. Tang W, Zeve D, Suh JM, Bosnakovski D, Kyba M, Hammer RE, Tallquist MD, Graff JM. White fat progenitor cells reside in the adipose vasculature. Science. 2008;322(5901):583-6.

3. Berry DC, Jiang Y, Graff JM. Emerging roles of adipose progenitor cells in tissue development, homeostasis, expansion and thermogenesis. Trends Endocrinol Metab: TEM. 2016;27(8):574-85.

4. Lockhart RA, Aronowitz JA, Dos-Anjos Vilaboa S. Use of freshly isolated human adipose stromal cells for clinical applications. Aesthet Surg J. 2017;37(suppl_3):S4-8.

5. Zimmerlin L, Donnenberg VS, Pfeifer ME, Meyer EM, Peault B, Rubin JP, Donnenberg AD. Stromal vascular progenitors in adult human adipose tissue. Cytometry A. 2010;77(1):22-30.

6. Mitterberger MC, Lechner S, Mattesich M, Kaiser A, Probst D, Wenger N, Pierer G, Zwerschke W. DLK1(PREF1) is a negative regulator of adipogenesis in CD105(+)/CD90(+)/CD34(+)/CD31(-)/FABP4(-) adipose-derived stromal cells from subcutaneous abdominal fat pats of adult women. Stem Cell Res. 2012;9(1):35-48.

7. Bertoli C, Skotheim JM, de Bruin RA. Control of cell cycle transcription during G1 and S phases. Nat Rev Mol Cell Biol. 2013;14(8):518-28.

8. Cristancho AG, Lazar MA. Forming functional fat: a growing understanding of adipocyte differentiation. Nat Rev Mol Cell Biol. 2011;12(11):722-34.

9. Tontonoz P, Spiegelman BM. Fat and beyond: the diverse biology of PPARgamma. Annu Rev Biochem. 2008:77:289-312.

10. Li D, Zhang F, Zhang X, Xue C, Namwanje M, Fan L, Reilly MP, Hu F, Qiang L. Distinct functions of PPARgamma isoforms in regulating adipocyte plasticity. Biochem Biophys Res Commun. 2016:481(1-2):132-8.

11. Farmer SR. Transcriptional control of adipocyte formation. Cell Metab. 2006;4(4):263-73.

12. Siersbaek R, Mandrup S. Transcriptional networks controlling adipocyte differentiation. Cold Spring Harb Symp Quant Biol. 2011;76:247-55.

13. Gentile AM, Lhamyani S, Coin-Araguez L, Oliva-Olivera W, Zayed H, Vega-Rioja A, Monteseirin J, Romero-Zerbo SY, Tinahones FJ, Bermudez-Silva FJ, et al. RPL13A and EEF1A1 are suitable reference genes for aPCR during adipocyte differentiation of vascular stromal cells from patients with different BMI and HOMA-IR. PLoS One. 2016;11(6):e0157002.

14. Bustin SA, Benes V, Garson JA, Hellemans J, Huggett J, Kubista M, Mueller R, Nolan T, Pfaffl MW, Shipley GL, et al. The MIQE guidelines: minimum information for publication of quantitative real-time PCR experiments. Clin Chem. 2009;55(4): 611-22.

15. Amable PR, Teixeira MV, Carias RB, Granjeiro JM, Borojevic R. Identification of appropriate reference genes for human mesenchymal cells during expansion and differentiation. PLoS One. 2013;8(9):e73792.

16. Dundas J, Ling M. Reference genes for measuring mRNA expression. Theory in biosciences $=$ Theorie in den Biowissenschaften. 2012;131(4):215-23.

17. Ferguson BS, Nam H, Hopkins RG, Morrison RF. Impact of reference gene selection for target gene normalization on experimental outcome using real-time qRT-PCR in adipocytes. PLoS One. 2010;5(12):e15208.

18. Andersen $\mathrm{CL}$, Jensen $\mathrm{J}$, Orntoft TF. Normalization of real-time quantitative reverse transcription-PCR data: a modelbased variance estimation approach to identify genes suited for normalization, applied to bladder and colon cancer data sets. Cancer Res. 2004;64(15):5245-50.

19. Julian GS, de Oliveira RW, Perry JC, Tufik S, Chagas JR. Validation of housekeeping genes in the brains of rats submitted to chronic intermittent hypoxia, a sleep apnea model. PloS one. 2014:9(10):e109902

20. Cairns RA, Harris IS, Mak TW. Regulation of cancer cell metabolism. Nat Rev Cancer. 2011;11(2):85-95. 
21. Tondeleir D, Vandamme D, Vandekerckhove J, Ampe C, Lambrechts A. Actin isoform expression patterns during mammalian development and in pathology: insights from mouse models. Cell Motil Cytoskeleton. 2009;66(10):798-815.

22. Arvidsson S, Kwasniewski M, Riano-Pachon DM, Mueller-Roeber B. QuantPrime--a flexible tool for reliable highthroughput primer design for quantitative PCR. BMC bioinformatics. 2008;9:465.

23. Vandesompele J, De Preter K, Pattyn F, Poppe B, Van Roy N, De Paepe A, Speleman F. Accurate normalization of realtime quantitative RT-PCR data by geometric averaging of multiple internal control genes. Genome Biol. 2002;3(7): RESEARCH0034.

24. Pfaffl MW, Tichopad A, Prgomet C, Neuvians TP. Determination of stable housekeeping genes, differentially regulated target genes and sample integrity: BestKeeper--excel-based tool using pair-wise correlations. Biotechnol Lett. 2004;26(6): 509-15.

25. Zwierzina ME, Ejaz A, Bitsche M, Blumer MJ, Mitterberger MC, Mattesich M, Amann A, Kaiser A, Pechriggl EJ, Horl S, et al. Characterization of DLK1(PREF1)+/CD34+ cells in vascular stroma of human white adipose tissue. Stem Cell Res. 2015; 15(2):403-18.

26. Ejaz A, Mitterberger MC, Lu Z, Mattesich M, Zwierzina ME, Horl S, Kaiser A, Viertler HP, Rostek U, Meryk A, et al. Weight loss upregulates the small GTPase DIRAS3 in human white adipose progenitor cells, which negatively regulates Adipogenesis and activates autophagy via Akt-mTOR inhibition. EBioMedicine. 2016;6:149-61.

27. Hellemans J, Mortier G, De Paepe A, Speleman F, Vandesompele J. qBase relative quantification framework and software for management and automated analysis of real-time quantitative PCR data. Genome Biol. 2007:8(2):R19.

28. Fink T, Lund P, Pilgaard L, Rasmussen JG, Duroux M, Zachar V. Instability of standard PCR reference genes in adiposederived stem cells during propagation, differentiation and hypoxic exposure. BMC Mol Biol. 2008;9:98.

29. Wong ML, Medrano JF. Real-time PCR for mRNA quantitation. BioTechniques. 2005;39(1):75-85.

30. Santos BP, da Costa Diesel LF, da Silva Meirelles L, Nardi NB, Camassola M. Identification of suitable reference genes for quantitative gene expression analysis in rat adipose stromal cells induced to trilineage differentiation. Gene. 2016;594(2): $211-9$

Ready to submit your research? Choose BMC and benefit from:

- fast, convenient online submission

- thorough peer review by experienced researchers in your field

- rapid publication on acceptance

- support for research data, including large and complex data types

- gold Open Access which fosters wider collaboration and increased citations

- maximum visibility for your research: over $100 \mathrm{M}$ website views per year

At $B M C$, research is always in progress.

Learn more biomedcentral.com/submissions 